- Madalena Maria Gomes de Queiróz Dias

Licenciada em Engenharia Quimica

Departamento de Engenharia Quimica da Universidade de Houston.

- José Alberto Nunes Ferreira Gomes

Licenciado em Engenharia Químico-Industrial

Institute of Molecular Biophysics - The Florida

State University.

- Emilia Alice dos Reis Torroaes Valente Licenciada em Engenharia Química
Departamento de Quimica da Universidade de Salford.

- Maria Manuela Regalo da Fonseca Licenciada em Engenharia Química Departamento de Engenharia Quimica da Universidade de Manchester - Instituto de Ciência e Tecnologia.

- José Duarte Ferreira Pedroso Botas Licenciado em Engenharia Química Departamento de Metalurgia de Sheffield.

\section{CONGRESSOS E CONFERÊNCIAS NO ESTRANGEIRO}

Alguns anúncios complementares aos que já sairam no Boletim anterior.

1981

Outubro

3

Pise

(Itália)

$11^{\text {th }}$ International Symposium on Clinical Pharmacology

\section{9 Montréal}

(Canadá)

$2^{\text {nd }}$ World Congress on Chemical Engineering

4-10 Detroit International Convention on the (Mich. USA) Water Pollution Control Federation

5. 8 Eger Symposium on Steric Effects in (Hungria)

5. 8 Szeged (Hungria)

6. 9 Bale (Suiça)

7. 9 Manilla (Filipinas)

$20-25$

Moscovo

Moscovo

$21 \cdot 24$

Singapura

\section{Novembro}

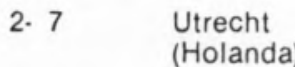

$16 \cdot 17$

$19 \cdot 20$

$6^{\text {th }}$ Colloquim on the Processing Technology and Technical Applications of Plastics

23-25

23.25 chmesse und Fachtagungen fur die Kerntechnische Industrie

$2^{\text {nd }}$ Asian International Chemical and Process Engineering (IUPAC)

$16 \cdot 17$

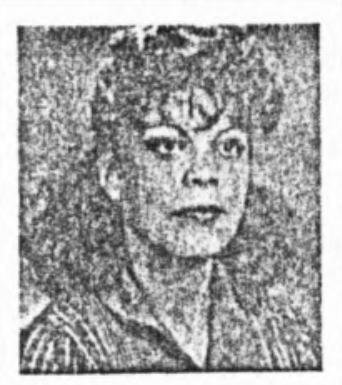

coordenaçāo

de M. Regina Tavares
RENERGIA 81 - Salon Professional international pour l'extraction, la génération, la maitrise et le recy. clage de l'énergie
Paris Journée d'étude "Rhéologie pour le França transformateur de thermoplasti. quesn

Londres International Conference on Fas (G.B.)

10.13 Bruxelas (Bélgica)

Miami Beach $2^{\text {nd }}$ International Symposium on. (FIa,USA)

18.19 Bruxelas (Bélgica)

iami Beact (FIa,USA)

Mulhouse (França)

Barcelona (Espanha)

Barcelone (Espanha)

Dezembro

Oxford

(G.B.)

$17 \cdot 18$

Linz

(Austria) Reactor Fuel Cycles

International Conference on Co balt, Metallurgy and Uses Environmental Pollution

Traitement de surface des métaux non ferreux

$2^{\text {nd }}$ International Chromatography Conference

Colloque national du G.F.P. "Sur face et interfaces dans les poly. mères"

$2^{\text {nd }}$ International Congress on Analytical Techniques in Environmen. tal Chemistry

$2^{\mathrm{e}}$ Congrès méditerranéen du Gé. nie chimique

Workshop on the "Chemistry and Analysis of Hydrocarbons in the Environment, 
1982

Março

$29 \cdot 2 / 4$

Swansea (G.-B.)

Chemistry of ions beams

Abril

$26 \cdot 30$

Lunteren (Holanda)

Surface ionization phenomena

Burgenstock Stereochemistry (Suiça)

Maio

$11 \cdot 14$

Ghent

(Bélgica)

Gregynog (G.-B.)

$24 \cdot 28$

Frejus (França)

Molten salts

$4^{\text {th }}$ International Symposium on Quantitative Mass Spectrometry in Life Sciences

Bio-Organic Chemistry

Junho

?

Estocolmo (Suécia)

Synthesis of low molecular weight carbohydrates" of biological signi.
Julho

$11 \cdot 16$

Louvain-la-neuve

(Bélgica)

18.23 Hull

(G.-B.)

Agosto

$?$

(Itália)

Setembro

$13 \cdot 17$

Londres (G.-B.)

27-1/10? Ferrara (Itália)

\section{Outubro}

$11 \cdot 14$

Lunteren (Holanda)

?(Outono) ? (R.F.A.) (Bélgica) ficance
Leuven (Bèlgica)
Sixth I.U.P.A.C. Conference on Phy. sical Organic Chemistry

Correlation analysis in organic che. mistry

Ionic Chemistry: Gaseous versus solvent ions

$14^{\text {th }}$ International Symposium on Cromatography

Pericyclic Reactions

Degradation processes in natural polymers

Short Living Species in Organic Chemistry and their Stabilization

Methods in Organic Chemistry

Magnetic Circular Dichroism

\section{ACTIVIDADES DA S.P.Q.}

Simpósio de Quimica Orgânica dedicado ao Professor Doutor António Jorge de Andrade Gouveia

Realiza-se na Faculdade de Ciências e Tecnologia da Universidade de Coimbra, Departamento de Quimica a 27 de Novembro de 1981, contando com a presença de Sir DEREK BARTON, prémio Nobel de Quimica, que proferirá a lição plenária.

Ficha de inscrição no Boletim n." 7.

Para informaçòes complementares contactar:

Prof. A. Rocha Gonçalves

Departamento de Química

Faculdade de Ciências e Tecnologia

Universidade de Coimbra

3000 COIMBRA

Telefones: 22826

$$
24207
$$

\section{$5{ }^{\circ}$ ENCONTRO NACIONAL DE QUÍMICA}

Porto - 29/Março a 3/Abril de 1982

\section{1 - COMISSÃO ORGANIZADORA}

Prof. J. J. Fraústo da Silva - Presidente da S.P.Q.

Prof. Rodrigues Guedes Carvalho - Fac. Engenharia (U.P.)

Prof. Francisco Carvalho Guerra - Fac. Farmácia (U.P.)

Prof. J. Barbosa Romero - Universidade do Minho Prof. A. J. C. Varandas - Fac. Ciências Tecnologia (U.C.)

Prof. Júlio Pedroso de Jesus - Universidade de Aveiro Prof. A. Romão Dias - secretário-geral da S.P.Q. Prof. M. A. V. Ribeiro da Silva - Delegação do Porto da S.P.Q.

Prof. Duarte J. V. Costa Pereira - Delegação do Porto da S.P.Q.

Dr. José L. F. C. Lima - Delegação do Porto da S.P.Q.

\section{2 - ORGANIZAÇÃO GERAL}

O Encontro terá a duração de uma semana e serí dividido em três blocos consecutivos; Investigaçâo, Indústria e Educação. 